\title{
Estado de coma y patrón electroencefalográfico de brote-supresión por sobredosis de Baclofeno.
}

\author{
Coma state and the electroencephalogram burst-suppression pattern related to baclofen overdose. \\ Juan Escobar-Montalvo ${ }^{1}$, Erika Herraez Sanchez 1, Emilia Petrica ${ }^{1}$, Ursula Ochoa Delgado De la Flor ${ }^{1}$
}

\section{RESUMEN}

El baclofeno es un análogo estructural del ácido gamma-aminobutírico empleado principalmente como relajante del músculo esquelético para el tratamiento de espasticidad y distonías. Las manifestaciones clínicas de intoxicación por este fármaco, se deben a alteración del nivel de conciencia que produce somnolencia, estado de coma e incluso la muerte. Desde el punto de vista electroencefalográfico, se ha descrito en estos casos, el llamado patrón de brotesupresión. Se presenta el caso de una mujer que súbitamente presentó dificultad para la bipedestación y mutismo acinético y, posteriormente, evolucionó a un estado de coma sin causa aparente. Una evaluación minuciosa en el Servicio de Emergencia, incluyó electroencefalograma urgente que resultó compatible con un patrón continuo de brote-supresión. Ante la sospecha de una intoxicación por baclofeno, la paciente fue tratada con diálisis, la cual revirtió el cuadro clínico y normalizó la actividad electroencefalográfica en un periodo de cinco días.

PALABRAS CLAVE: Baclofeno, brote-supresión, coma.

\section{SUMMARY}

Baclofen is a structural analog of gamma-aminobutyric acid, primarily used as a skeletal muscle relaxant for treatment of spasticity and dystonia. The clinical picture of baclofen intoxication includes reduction or loss of consciousness and manifestations such as sleepness, coma or even death. Electroencephalograms performed in these cases have consistently shown the so-called burst-suppression pattern. The case of a female patient who suddenly presented difficulty to still-standing and mutism and later evolved into coma, is presented. A detailed study was performed in the emergency department, including an electroencephalogram compatible with a continuous burst-suppression pattern. The patient was treated with dialysis in the face of a possible baclofen intoxication and, in a period of five days, the clinical symptoms reverted and the electroencephalographic activity was normalized.

KEYWORDS: Baclofen, burst-suppression, coma.

\section{INTRODUCCIÓN}

El baclofeno es un análogo estructural del ácido gamma-aminobutírico (GABA) usado como relajante del músculo esquelético para el tratamiento de la espasticidad y las distonías. En el sistema nervioso central (SNC) actúa como depresor posiblemente por ser un agonista de los receptores GABA-B (1-10).
Los efectos adversos descritos de la intoxicación por baclofeno son variados e incluyen hipotensión arterial, bradicardia, apneas centrales, crisis convulsivas, Status epilepticus no convulsivo, somnolencia, estupor, coma e incluso la muerte (11-17). Desde el punto de vista electroencefalográfico se ha descrito el patrón de brote-supresión en algunos pacientes con intoxicación por baclofeno (18-21). Presentamos

Sección de Neurofisiología Clínica, Servicio de Neurología del Hospital Universitario La Paz. Madrid, España. 
Estado de coma y patrón electroencefalográfico de brote-supresión por sobredosis de Baclofeno.

el caso de una paciente que tras la sobreingesta de baclofeno presentó un estado de coma asociado a un patrón electroencefalográfico de brote-supresión.

\section{PRESENTACIÓN DEL CASO}

Presentamos a una mujer de 18 años de edad, extranjera con 8 meses de tiempo de residencia en España, sin antecedentes patológicos conocidos; que fue encontrada en su habitación con dificultad para mantener la bipedestación y mutismo acinético. Estos síntomas progresaron a los pocos minutos a disminución del nivel de conciencia, pérdida del tono muscular y ausencia de respuesta a estímulos. Fue trasladada al Servicio de Urgencias de un Hospital General donde a pesar de tener las funciones vitales y los valores de saturación de oxígeno en rangos de normalidad, persistía con un nivel de conciencia compatible con un estado de coma (puntaje en la escala de Glasgow $=3$ puntos). En la evaluación inicial presentaba pupilas mióticas, isocóricas y arreactivas, ausencia de reflejos oculocefálico, tusígeno y de liberación piramidal, ausencia de respuesta motora a estímulos sensitivos y dolorosos, hipotonía y arreflexia generalizadas. Debido a esta sintomatología se le realizó una intubación endotraqueal (no fue necesaria la utilización de propofol) con la finalidad de mantener permeable la vía aérea y posteriormente fue trasladada a una unidad de vigilancia intensiva.

Los resultados de los estudios analíticos en sangre fueron normales para hemograma, tiempos de coagulación, dímero $\mathrm{D}$, glucosa, urea, creatinina, iones (calcio, sodio, magnesio, potasio, cloro y fósforo), lactato deshidrogenasa, proteína $\mathrm{C}$ reactiva, hormona estimulante de la tiroides y tiroxina. El estudio del líquido cefalorraquídeo no presentó hematíes ni pleocitosis, la tinción de gram fue negativa, el cultivo fue estéril y los niveles de glucosa, proteínas, lactato, adenosindeaminasaestuvieronenrangosdenormalidad. El análisis del sedimento urinario fue normal. No se detectaron trazas en orina de anfetaminas, cocaína, cannabis, metanfetaminas, opiáceos, benzodiacepinas, antidepresivos tricíclicos, barbitúricos, ni metadona. El registro electrocardiográfico y la radiografía de tórax fueron normales.

Ante la posibilidad de un evento cerebral de etiología vascular aguda o tumoral se realizaron una tomografía craneal computarizada y una angiografía cerebral urgentes que no mostraron lesiones ocupantes de espacio ni obstrucciones en las arterias del polígono de Willis, ni en los ejes carotideos ni vertebrales.
Al persistir en estado de coma, a pesar que los resultados analíticos y radiológicos fueron normales, y por la sospecha de un status epilepticus no convulsivo, se solicitó un electroencefalograma (EEG) urgente de 30 minutos de duración. Los hallazgos del EEG mostraron una actividad bioeléctrica cerebral polimórfica constituida por brotes síncronos, simétricos y bihemisféricos, de ondas delta a $0.5 \mathrm{~Hz}$ de frecuencia y $60-80 \mu \mathrm{V}$ entremezcladas con puntas/ ondas agudas de entre $100-200 \mu \mathrm{V}$ de amplitud y de 0.3-0.6 ms de duración. Estos brotes se alternaban con periodos de entre 2-5 segundos de duración de muy baja amplitud (amplitud menor de $10 \mu \mathrm{V}$ ) o supresión completa de la actividad eléctrica cerebral (figura 1). Este patrón se observó en el 100\% del registro.

Tras este hallazgo electroencefalográfico y ante la sospecha de una intoxicación aguda se interrogó nuevamente a los familiares. El padre refirió que su esposa se encontraba en tratamiento con baclofeno $(10 \mathrm{mg} / 8$ horas $)$ por un cuadro de espasticidad secundario a una paraplejia post-traumática. Asimismo, refirió haber encontrado un paquete vacío de 10 comprimidos de baclofeno, de 10 miligramos, en la habitación de la paciente, no refiriendo la posibilidad de otra sustancia potencialmente tóxica. Tras obtener esta información se intentó realizar la medición de niveles de baclofeno en sangre y/o líquido cefalorraquídeo, sin embargo, en el hospital donde fue atendida no se realizaba esta determinación analítica.

Ante la alta sospecha clínica de una intoxicación por baclofeno, se le realizaron 2 sesiones de hemodiálisis, siguiendo un protocolo de diálisis para intoxicaciones farmacológicas agudas. Tras la realización de la segunda diálisis, la paciente presentó una mejoría progresiva del nivel de conciencia y de la fuerza muscular. A las 24 horas de permanecer en la Unidad de Vigilancia Intensiva y habiendo presentado una normalización del nivel de conciencia (escala de Glasgow de 15 puntos) y mejoría de la fuerza muscular, fue trasladada a un Servicio de Medicina Interna.

Durante este ingreso hospitalario, fue evaluada por especialistas en psiquiatría, a quienes les refirió que, tras discutir con su hermana mayor, y sin planificarlo ingirió un envase de pastillas de baclofeno. Además, refirió tener un ánimo bajo por la situación que vive su madre y por dificultades de adaptación a un nuevo entorno social. El cuadro clínico fue catalogado como un trastorno adaptativo con predominio depresivo en el contexto de inmigración y accidente de un familiar de primer grado. Se le indicó tratamiento antidepresivo 


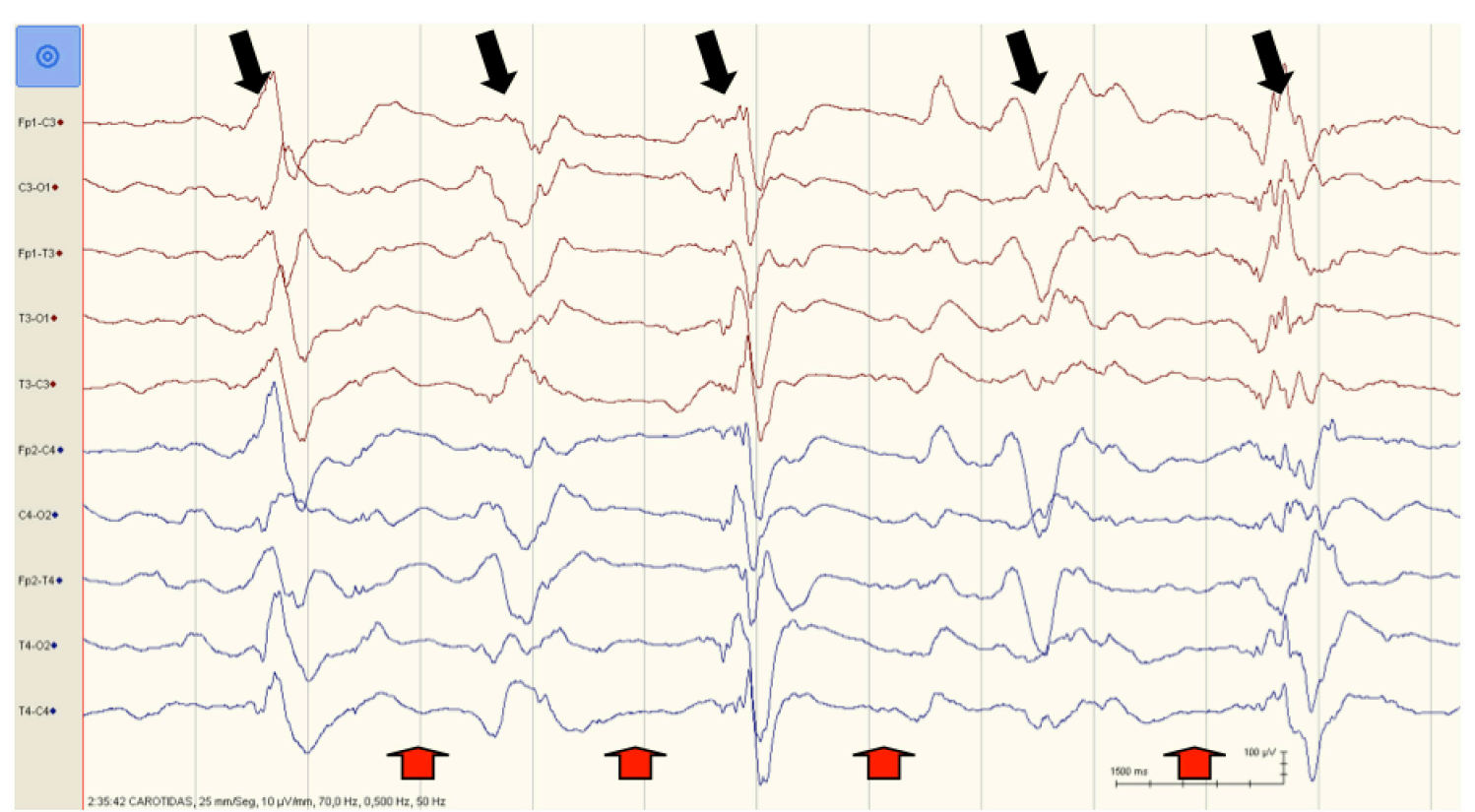

Figura 1. Patrón electroencefalográfico de brote-supresión encontrado en la paciente. Flechas negras: brote. Flechas rojas: supresión.

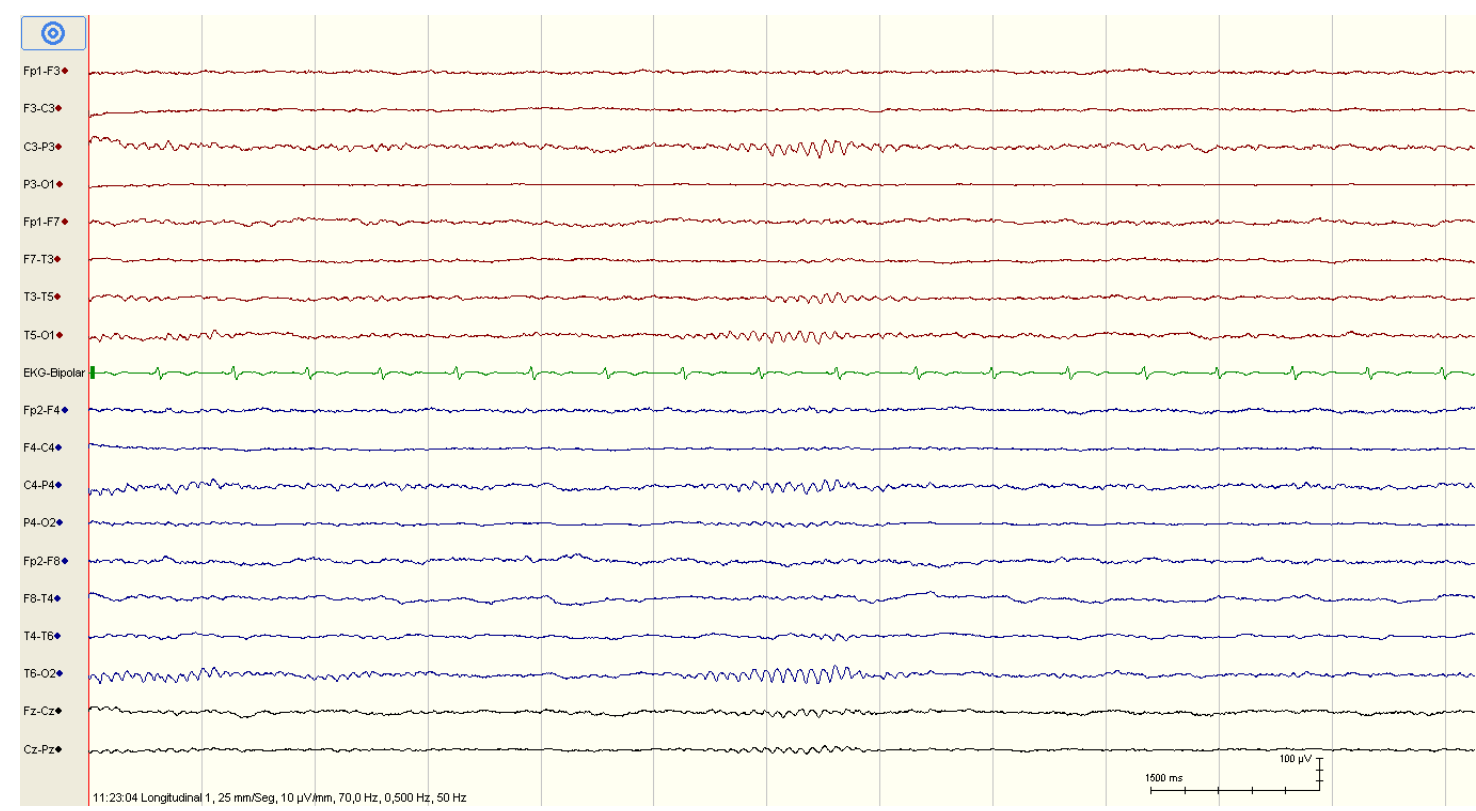

Figura 2. EEG de características normales. Ritmo dominante parieto-occipital en rango alfa y ritmos rápidos en rango beta en regiones cerebrales anteriores.

$\mathrm{y}$ ansiolítico, y seguimiento en las consultas de psiquiatría tras el alta hospitalaria.

Previamente al alta, tras 5 días de ingreso hospitalario, y estando con una recuperación completa del nivel de conciencia, de la fuerza muscular y mejoría del estado de ánimo, se le realizó un segundo EEG que fue de características normales para la edad de la paciente (figura 2).

\section{DISCUSIÓN}

El baclofeno (ácido 4-amino-3-[4-clorofenil]butanoico) es un análogo estructural del GABA de acción inhibitoria que es usado como relajante del músculo esquelético $(1,2)$. Sus principales indicaciones son para el tratamiento de las distonías y de la espasticidad (3-5). Otras indicaciones incluyen el tratamiento del reflujo gastroesofágico, rumiación, hipo 
Estado de coma y patrón electroencefalográfico de brote-supresión por sobredosis de Baclofeno.

recurrente, disfunción vesical, neuralgia del trigémino y dependencia alcohólica (6-10). Desde el punto de vista farmacocinético, al ser una molécula lipofílica, tiene una absorción gastrointestinal rápida con un pico plasmática de 1-3 horas tras la ingestión, atraviesa la barrera hematoencefálica en concentraciones equivalentes al $12 \%$ de los niveles plasmáticos y tiene un tiempo de vida media de 3-7 horas en el plasma y 5 horas en el líquido céfalorraquídeo. Estos valores de vida media se pueden incrementar hasta 35 horas en casos de intoxicación. La eliminación es en un 75\%$80 \%$ renal y $15 \%$ hepática (22-24).

El en el sistema nervioso central (SNC) tiene efecto depresor, posiblemente por ser un agonista del GABA-B lo que condiciona una reducción de la liberación de los neurotransmisores excitatorios de aspartato y glutamato (25-27). Dentro de los principales efectos adversos secundarios a la intoxicación (oral o intratecal) por Baclofeno se han descrito mareos, somnolencia, aturdimiento, apneas centrales, hipotensión arterial, bradicardia, crisis convulsivas, mutismo acinético, estupor, coma e incluso la muerte (11-17). Además de estas manifestaciones clínicas se ha descrito el patrón electroencefalográfico de brote-supresión secundario a la intoxicación con baclofeno (18-21). De manera general, este patrón se encuentra habitualmente asociado al estado de coma. La gravedad y pronóstico asociados al brotesupresión en pacientes en estado de coma dependen directamente de su etiología subyacente. Las entidades de mal pronóstico asociadas con este patrón son la encefalopatía post-anóxica posterior a una parada cardio-respiratoria, las encefalopatías neonatales como el síndrome de Otahara, los traumatismos encefálicos graves, tumores cerebrales entre otras causas. Los pronósticos menos graves o reversibles son principalmente de etiología farmacológica (intoxicaciones con barbitúricos, inducción anestésica con Propofol, tratamiento con ketamina, entre otros) (11-17, 28). Electroencefalográficamente, el patrón de brote-supresión se define por la presencia de brotes hipervoltados $(>50 \mu \mathrm{V})$ de actividad cerebral que se alternan por periodos de supresión/atenuación de actividad eléctrica cerebral. Los brotes se conforman por ondas en rango theta/delta de voltaje variable que se entremezclan con ondas agudas/puntas de entre 50$1000 \mu \mathrm{V}$ y que tienen una duración de entre 100-1000 ms. Estos brotes se entremezclan con periodos de supresión (amplitud $<10 \mu \mathrm{V}$ ) o atenuación (amplitud $<20 \mu \mathrm{V}$ ) de la actividad eléctrica cerebral de duración variable. Habitualmente se caracterizan por ser síncronos, simétricos y bihemisféricos. Sin embargo, pueden ser unilaterales en casos de lesiones focales (29-31)

Otros hallazgos electroencefalográficos descritos en paciente con intoxicación por Baclofeno son la lenificación de la actividad de fondo, el incremento de ondas theta, aparición de ondas agudas y puntas que pueden asociarse con crisis epilépticas y/o Status epilepticus (vía intratecal principalmente), aparición de ondas trifásicas relacionadas con encefalopatía metabólica, ondas delta generalizadas, y atenuación/ supresión completa de la actividad cerebral (32-35).

En el presente caso se describe a una mujer quien tras la sobreingesta de baclofeno presentó un estado de coma y patón electroencefalográfico de brote-supresión, que revirtieron tras el tratamiento con hemodiálisis. A diferencia de algunos reportes previamente descritos, en este caso no se pudo confirmar la intoxicación farmacológica, debido a la falta de cuantificación de los niveles de Baclofeno en suero y/o en líquido cefalorraquídeo, por no haberse tenido la factibilidad para medir este fármaco de forma urgente en el hospital en que fue atendida la paciente. Sin embargo, a pesar de la falta de determinación de estos niveles, es coherente pensar que se trató de un caso de coma inducido por Baclofeno, porque la paciente presentó un cuadro clínico de debilidad muscular y disminución del nivel de conciencia que progresó hasta el coma, por presentar un patrón electroencefalográfico de brote supresión, por la normalidad de las otras pruebas auxiliares solicitadas y porque tras estar recuperada refirió haber ingerido este fármaco previamente a la aparición de los síntomas.

Finalmente, un aspecto fundamental a destacar fue la importancia que tuvo en el proceso diagnóstico la realización de una anamnesis detallada y exhaustiva, enfatizando sobre la posibilidad de una etiología farmacológica como la causante del cuadro electroclínico de la paciente. Esta indagación permitió actuar de manera oportuna realizando un tratamiento precoz, resolviendo el cuadro clínico y mejorando el pronóstico vital de la paciente. Además, a pesar de ser una prueba auxiliar, se destaca importancia que tuvo en el proceso diagnóstico la realización de un electroencefalograma urgente.

\section{Correspondencia}

Juan Escobar-Montalvo

Correo electrónico: juanma_05@hotmail.com 


\section{REFERENCIAS BIBLIOGRÁFICAS}

1. Brogden RN, Speight TM, Avery GS. Baclofen: A preliminary report of its pharmacological properties and therapeutic efficacy in spasticity. Drugs. 1974; $8(1): 1-14$. DOI: $\quad 10.2165 / 00003495-197408010-$ 00001

2. Milanov IG. Mechanisms of baclofen action on spasticity. Acta Neurol Scand. 1992;85;(5):305-10.

3. Rekand T, Grønning M. Treatment of spasticity related to multiple sclerosis with intratechal baclofen: A long-term follow-up. J Rehabil Med. 2011; 43(6):511-4. doi: 10.2340/16501977-0811.

4. Hudgson $\mathrm{P}$, Weightman D. Baclofen in thetreatment of spasticity. Br Med J. 1971; 4(5778):15-17. DOI: 10.1136/bmj.4.5778.15

5. Hedley DW, Maroun JA, Espir ML. Evaluation of baclofen (Lioresal) for spasticity in multiple sclerosis. Postgraduate Medical Journal. 1975;51:615-618. DOI: $10.1136 /$ pgmj.51.599.615

6. Rozatkar AR, Kapoor A, Sidana A, Chavan BS. Clinical experience of baclofen in alcohol dependence: A chart review. Ind Psychiatry J. 2016; 25(1):11-16. doi: 10.4103/0972-6748.196043.

7. Agabio R, Preti A, Gessa G. Efficacy and tolerability of baclofen in substance use disorders: A Systematic Review. Eur Addict Res. 2013;19(6):325-45. doi: $10.1159 / 000347055$

8. Garbutt JC, Flannery B. Baclofen for alcoholism. Lancet. 2007;370(9603):1884-5. DOI: 10.1016/ S0140-6736(07)61789-9

9. Blondeau K, Boecxstaens V, Rommel N, Farré R, Depeyper S, Holvoet L, et al. Baclofen improves symptoms and reduces postprandial flow events in patients with rumination and supragastric belching. Clin Gastroenterol Hepatol. 2012;10(4):379-84.

10. Li S, Shi S, Chen F, Lin J. The effects of baclofen for the treatment of gastroesophageal reflux disease: A meta-analysis of randomized controlled trials. Gastroenterol Res Pract. 2014;2014:307805. doi: $10.1155 / 2014 / 307805$

11. Olivier PY, Joyeux-Faure M, Gentina T, Launois SH, d'Ortho MP, Pépin JL, et al. Severe central sleep apnea associated with chronic baclofen therapy A case series. Chest. 2016;149(5):127-131.

12. Radhakrishnan H. Baclofen-induced neurotoxicity in a patient with end-stage renal disease. Saudi J Kidney Dis Transpl. 2016;27(3):595-597.

13. Ostermann ME, Young B, Sibbald WJ, Nicolle MW. Coma mimicking brain death following baclofen overdose. Intensive Care Med. 2000;26(8):1144-6.

14. Anderson KJ, Farmer JP, Brown K. Reversible coma in children after improper baclofen pump insertion. Paediatr Anaesth. 2002;12(5):454-60.

15. Alonso-Navarro H, Adeva-Bartolomé M, MartínPrieto M, Ruiz-Ezquerro J, Jiménez-Jiménez J. Coma inducido por sobredosis de baclofeno intratecal. Rev Neurol. 2006;43(1):63-4.

16. Caron E, Morgan R, Wheless JW. An unusual cause of flaccid paralysis and coma: baclofen overdose. $\mathrm{J}$ Child Neurol. 2014:29(4):555-9.

17. Sullivan R, Hodgman M, Kao L, Tormoehlen L. Baclofen overdose mimicking brain death. Clinical Toxicology. 2012; 50: 141-144.

18. Montes MI, Hernández Ortiz OH, Guerra-Palacio A. Electroencephalographicburst-suppressionpatterndue to baclofen intoxication. Med Intensiva. 2011;35 (9):584-6.

19. Weissenborn K, Wilkens H, Hausmann E, Degen PH. Burst suppression EEG with baclofen overdose. Clin Neurol Neurosurg. 1991;93(1):77-80.

20. Boutte C, Vercueil L, Durand M, Vincent F, Alvarez JC. EEG contribution to the diagnosis of baclofen overdose. Neurophysiol Clin. 2006; 36(2):85-9.

21. Weissenborn K, Wilkens H, Hausmann E, Degen PH. Burst suppression EEG with baclofen overdose. Clin Neurol Neurosurg. 1991;93(1):77-80.

22. Wuis EW, Dirks MJM, Termind EFS, Vree TB, Van der Kleijn E. Plasma and urinary excretion kinetics of oral Baclofen in healthy subjects. Eur J Clin Pharmacol. 1989;37:181-184.

23. Sullivan R, Hodgman MJ, Kao L, Tormoehlen LM. Baclofen overdose mimicking brain death. Clin Toxicol (Phila). 2012;50:141-4.

24. Ghose K, Holmes KM, Matthewson K. Complications of baclofen overdosage. Postgrad Med J. 1980;56:865.

25. Kantamneni S. Cross-talk and regulation between glutamate and GABAB receptors. Frontiers in Cellular Neuroscience. 2015;9(135):1-7.

26. Geng Y, Bush M, Mosyak L, Wang Feng, Fan Q. Structural mechanism of ligand activation in human GABAB receptor. Nature. 2013;(504):254-259.

27. Zhang J, Shen W, Slaughter M. Two metabotropic $\gamma$-aminobutyric acid receptors differentially modulate calcium currents in retinal ganglion cells. J Gen Physiol. 1997;110(1):45-58.

28. Hofmeijer J, Tjepkema-Cloostermans MC, van Putten MJ. Burst-suppression with identical bursts: a distinct EEG pattern with poor outcome in postanoxic coma. Clin Neurophysiol. 2014;125(5):947-954.

29. Amzica f. What does burst suppression really mean? Epilepsy \& Behavior. 2015; 49 (2015):234-237. doi: 10.1016/j.yebeh.2015.06.012

30. Amzica F. Basic physiology of burst-suppression.

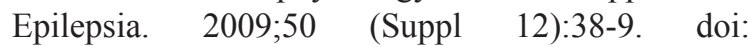
10.1111/j.1528-1167.2009.02345.x

31.Niedermeyer M, Sherman D, Geocadin R, Hansen HC, Hanley D. The Burst-Suppression Electroencephalogram. J Clinical Electroencephalography. 1999; 30 (3): 99-105. Doi: https://doi.org/10.1177/155005949903000305 
Estado de coma y patrón electroencefalográfico de brote-supresión por sobredosis de Baclofeno.

32. Hirsch LJ, LaRoche SM, Gaspard N, Gerard E, Svoronos A, Herman ST, et al. American Clinical Neurophysiology Society's Standardized Critical Care EEG Terminology: 2012 version. J Clin Neurophysiol. 2013;30(1):1-27. DOI: 10.1097/ WNP.0b013e3182784729

33. Badr GG, Matousek M, Frederiksen PK. A quantitative EEG analysis of the effects of baclofen on man. Neuropsychobiology. 1983;10(1):13-8. DOI: 10.1159/000117978
34. Wainapel S, Lee L, Riley T. Reversible electroencephalogram changes associated with administration of baclofen in a quadriplegic patient: Case Report. Paraplegia. 1986; 24(2):123-6. DOI: 10.1038/sc. 1986.16

35. Miller J. Baclofen overdose mimicking anoxic encephalopathy: a case report and review of the literature. Ther Adv Drug Saf. 2017;8(5):165-167. doi: $10.1177 / 2042098617693571$

Recibido: 21/05/2019

Aceptado: 19/06/2019 